\title{
Temperature Dependency of Schottky Barrier Parameters of Ti Schottky Contacts to Si-on-Insulator
}

\author{
I. Jyothi ${ }^{1}$, Hyun-Deok Yang ${ }^{1}$, Kyu-Hwan Shim ${ }^{1}$, V. Janardhanam ${ }^{2}$, \\ Seung-Min $\mathrm{Kang}^{3}$, Hyobong Hong ${ }^{4}$ and Chel-Jong Choi ${ }^{1,2, *}$ \\ ${ }^{1}$ School of Semiconductor and Chemical Engineering, Semiconductor Physics Research Center (SPRC), \\ Chonbuk National University, Jeonju 561-756, Korea \\ ${ }^{2}$ Department of BIN Fusion Technology, Chonbuk National University, Jeonju, 561-756, Republic of Korea \\ ${ }^{3}$ Department of Advanced Materials Science and Engineering, Hanseo University, Seosan 360-706, Republic of Korea \\ ${ }^{4}$ IT Convergence Technology Research Laboratory, Electronics \& Telecommunication Research Institute, \\ Daejeon, 305-700, Republic of Korea
}

\begin{abstract}
We have investigated the temperature-dependent current-voltage $(I-V)$ characteristics of Ti Schottky structure on the Si-on-insulator (SOI) in the temperature range of $175-375 \mathrm{~K}$ by steps of $25 \mathrm{~K}$. As decreasing temperature, the barrier height and ideality factor of Ti/SOI Schottky contact were found to be decreased and increased, respectively, indicating a considerable deviation from the ideal thermionic emission model in its current conduction mechanism. From the linear relationship between the barrier heights and ideality factors, the homogeneous barrier height was calculated to be $0.76 \mathrm{eV}$. The mean barrier height of $0.87 \mathrm{eV}$ and the modified Richardson constant value of $30.63 \mathrm{~A} \cdot \mathrm{cm}^{-2} \cdot \mathrm{K}^{-2}$ were obtained using modified Richardson plot. On the basis of a thermionic emission mechanism with a Gaussian distribution of the barrier heights, the temperature-dependent $I-V$ behavior of Ti/SOI Schottky contact was explained in terms of barrier height inhomogeneities at the interface between Ti and SOI. [doi:10.2320/matertrans.M2013015]
\end{abstract}

(Received January 11, 2013; Accepted June 5, 2013; Published July 19, 2013)

Keywords: silicon-on-insulator, titanium, Schottky contact, Schottky barrier inhomogeneities, Gaussian distribution

\section{Introduction}

Over the past few decades, metal-semiconductor Schottky structures play an important role in the microelectronics industry. ${ }^{1-3)}$ In particular, the fabrication of reliable and well-controlled Schottky contacts are essential for successful device operation. Until now, Ti has been widely used as a contact material for the practical device application due to its excellent adhesion on Si surface and low work function that promotes the formation of high barrier height on p-type $\mathrm{Si}^{4}{ }^{4}$ With the ever-increasing demand for the continuous scalingdown of complementary metal oxide semiconductor (CMOS) devices, Si-on-insulator (SOI) devices have been on demand due to their advantages such as lower parasitic capacitance, better sub threshold characteristics, superior electrical isolation and immunity to short-channel effects over conventional bulk Si ones. ${ }^{5-8)}$ As considering the prominent features of SOI, the fabrication of Ti Schottky contact to SOI can be expected to offer the promise of realizing high performance device. Moreover, a detailed knowledge about the electrical characteristics of Ti/SOI Schottky contact is substantially important for further improvement of device performance. Generally, the analysis of the current-voltage $(I-V)$ characteristics of Schottky contacts obtained only at room temperature does not give complete information about the carrier transport across the Schottky barrier and about the nature of the Schottky barrier formed at the metal-semiconductor interface. In fact, it neglects many possible effects that cause non-ideality in the $I-V$ characteristics. On the other hand, the temperature-dependent $I-V$ characteristics gives a better understanding of the conduction mechanism and to under-

*Corresponding author, E-mail: cjchoi@chonbuk.ac.kr stand different aspects that shed light on the validity of various processes involved. ${ }^{9)}$ In the present work, we fabricated the Ti Schottky contact to p-type SOI, and evaluated its electrical properties. Especially, temperaturedependent $I-V$ characteristics were performed and it will be shown that based on the assumption of the Gaussian distribution of barrier heights, the inhomogeneities of barrier height at the interface are responsible for the temperature dependency of Schottky barrier parameters of Ti/SOI Schottky contact.

\section{Experimental Details}

The p-type (100) UNIBOND SOI wafers with a resistivity of $10 \Omega \cdot \mathrm{cm}$ were used as a starting material. The thicknesses of the top $\mathrm{Si}$ and buried oxide (BOX) layers were 100 and $200 \mathrm{~nm}$, respectively. Initially, the SOI wafers were cleaned in an ultrasonic bath of acetone and methanol, immersed in a diluted HF solution $\left(\mathrm{H}_{2} \mathrm{O}: \mathrm{HF}=100: 1\right)$ to remove the native oxide and finally rinsed with de-ionized (DI) water. To form Schottky contacts, $30 \mathrm{~nm}$-thick Ti films were deposited by means of radio-frequency magnetron sputtering at room temperature. The circular Ti Schottky contacts were defined with a diameter of $200 \mu \mathrm{m}$, around which Ohmic contacts were formed by the sputter deposition of 50-nm thick Ni films separated by a distance of $100 \mu \mathrm{m}$. Generally, since $\mathrm{Ni}$ has a high work function $(5.15 \mathrm{eV})$, it has been widely employed to form Ohmic contact to p-type semiconductor. ${ }^{10,11)}$ For a comparison, Ti Schottky contacts to p-type bulk Si were fabricated using same process conditions. The temperature-dependent $I-V$ measurements were performed using a precision semiconductor parameter analyzer (Agilent 4156C). 


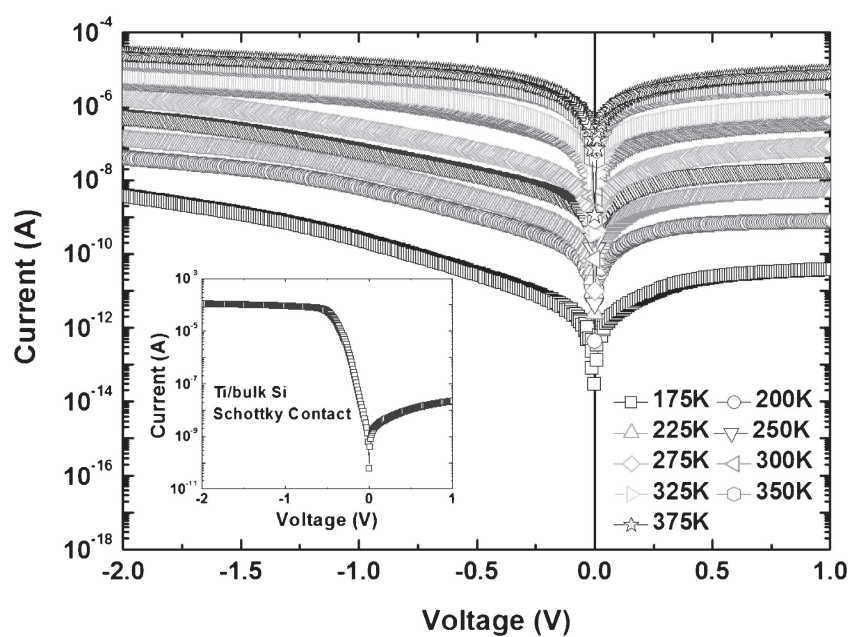

Fig. 1 Temperature-dependent $I-V$ characteristics of Ti/SOI Schottky contact in the temperature range of $175-375 \mathrm{~K}$. Inset shows $I-V$ characteristics of $\mathrm{Ti} /$ bulk $\mathrm{Si}$ Schottky contact measured at room temperature.

\section{Results and Discussion}

The $I-V$ characteristics of Ti/SOI Schottky contacts are evaluated as a function of forward and reverse bias conditions at temperature interval $(175-375 \mathrm{~K})$ and illustrated in Fig. 1. The Schottky diode parameters such as barrier height and ideality factor are analyzed only in the forward bias region of $I-V$ characteristics as considering the field dependence effects of the carrier transport under reverse bias condition. When the carrier transport governed by thermionic emission, the current through the barrier of a Schottky contact (from the semiconductor into the metal) is given by ${ }^{12-14)}$

$$
I=I_{0} \exp \left(\frac{q V}{n k T}\right)\left[1-\exp \left(\frac{-q V}{k T}\right)\right]
$$

where $V$ is the definite forward-bias voltage across the junction, $q$ is the electronic charge, $k$ is the Boltzmann's constant and $T$ is the absolute temperature in Kelvin and $I_{0}$ is the saturation current ensuing from the straight line intercept of the $\ln I-V$ plot at $V=0$ and is expressed as ${ }^{13,15)}$

$$
I_{0}=A A^{*} T^{2} \exp \left(\frac{-q \Phi_{\text {bo }}}{k T}\right)
$$

where, $A$ is the diode area, $A^{*}$ is the Richardson constant of $32 \mathrm{~A} \cdot \mathrm{cm}^{-2} \cdot \mathrm{K}^{-2}$ for $\mathrm{p}$-type $\mathrm{Si}^{16)} \Phi_{\mathrm{bo}}$ is the zero bias effective barrier height and $n$ is the ideality factor which is a measure of conformity of the diode to pure thermionic emission. The ideality factor is obtained from the slope of straight line portion of the forward bias $\ln I-V$ characteristics from eq. (1) and it can be written as

$$
n=\frac{q}{k T}\left(\frac{\mathrm{d} V}{\mathrm{~d}(\ln I)}\right)
$$

From eq. (2), the barrier height is given by

$$
\Phi_{\mathrm{bo}}=\frac{k T}{q} \ln \left(\frac{A A^{*} T^{2}}{I_{0}}\right)
$$

The values of ideality factor and barrier height calculated using eqs. (3) and (4), respectively are summarized in Fig. 2.

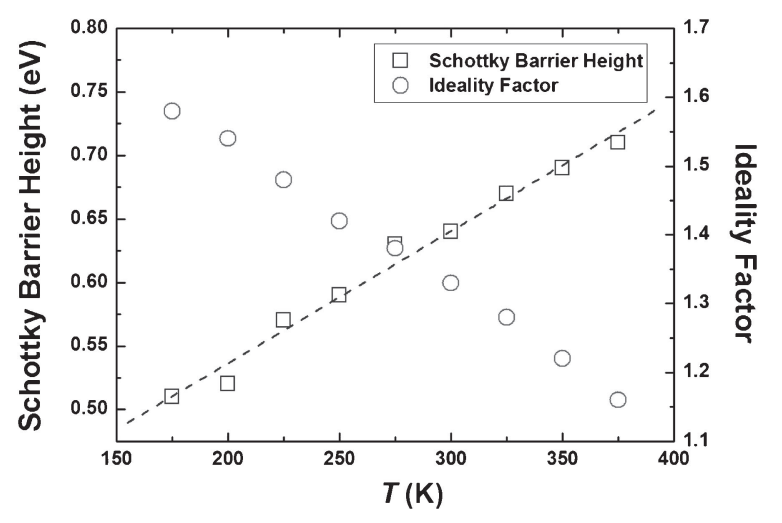

Fig. 2 Plot of barrier height and ideality factor extracted from Ti/SOI Schottky contact as function of temperatures in the range of $175-375 \mathrm{~K}$.

It is clear that from $I-V$ characteristics at the room temperature, Ti Schottky contacts to SOI exhibits worse Schottky-barrier behavior than Ti/bulk Si Schottky contacts (the inset of Fig. 1). For instance, the barrier heights are calculated to be 0.64 and $0.70 \mathrm{eV}$ for Ti Schottky contacts to $\mathrm{SOI}$ and bulk $\mathrm{Si}$, respectively. Furthermore, the ideality factor of Ti/bulk Si Schottky contact is 1.12 whereas the Ti/SOI Schottky contact shows a higher value of ideality factor being 1.33. It should be noted that the barrier height and ideality factor extracted from Ti/bulk Si Schottky contact are comparable to previously reported values by Ohta et al. ${ }^{4)}$ When considering Ti/bulk $\mathrm{Si}$ and Ti/SOI Schottky contacts, they are fabricated using same process conditions, the degraded $I-V$ behavior of Ti Schottky contacts to SOI seems not to be associated with process damages occurring during device manufacturing. On the other hand, the inferior performance of Ti/SOI Schottky contact relative to the Ti/bulk Si Schottky contact could be attributed to the quality of SOI film. Unlike to conventional Si wafers, the SOI wafers have more metallic contamination, structural defects and surface roughness in the top Si layer which might be introduced during the fabrication process of SOI wafers. ${ }^{17,18)}$ It seems that these imperfections of SOI wafers could be a main cause of the barrier height inhomogeneity, which makes the $I-V$ characteristic of Ti/SOI Schottky contact worse as compared to the Ti/bulk Si Schottky contact. Non-ideal $I-V$ characteristics of Ti/SOI Schottky contact shown in Fig. 1 implies the deviation from pure thermionic emission theory. Theoretically, the barrier heights and ideality factors obtained from ideal $I-V$ characteristics governed by pure thermionic emission should be independent of temperature.

The barrier height can be determined in another way by using the Richardson plot of $\ln \left(I_{0} / T^{2}\right)$ versus $1000 / T$, as shown in Fig. 3. The eq. (2) can be rewritten as

$$
\ln \left(\frac{I_{0}}{T^{2}}\right)=\ln \left(A A^{*}\right)-\frac{q \Phi_{\text {bo }}}{k T}
$$

According to eq. (5), the plot should yield a straight line with the intercept yielding the Richardson constant. The slope yields the activation energy, if the Schottky barrier $\Phi_{\text {bo }}$ is independent of temperature $T$. The bowing of the curve demonstrates that it is impossible to fit our data with a temperature-independent barrier $\Phi_{\mathrm{bo}}$. Therefore, the barrier 


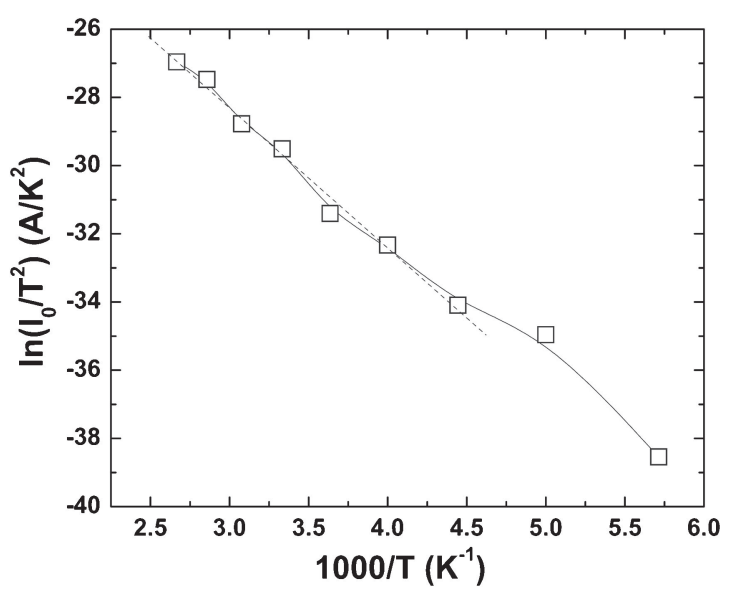

Fig. 3 Richardson plot of $\ln \left(I_{0} / T^{2}\right)$ versus $1000 / \mathrm{T}$ of for Ti/SOI Schottky contact.

height $\left(\Phi_{\mathrm{bo}}\right)$ values were determined directly from the $I_{0}$ values with the help of eq. (4) and a Richardson constant $A^{*}=32 \mathrm{~A} / \mathrm{cm}^{2} \mathrm{~K}^{2}$, for p-type $\mathrm{Si}$. The results of temperature dependent barrier height $\Phi_{\mathrm{bo}}(T)$ are shown in Fig. 2 together with values for the temperature dependent ideality factor $n(T)$ respectively. Activation energy value of $0.35 \mathrm{eV}$ was obtained from the slope of this straight line for the Ti/SOI Schottky barrier diode. The Richardson constant calculated from the intercept of the straight line portion at the ordinate of the experimental $\ln \left(I_{0} / T^{2}\right)$ versus $1000 / T$ plot in Fig. 3 is found to be $3.5 \times 10^{-4} \mathrm{~A} \cdot \mathrm{cm}^{-2} \cdot \mathrm{K}^{-2}$, of which the value is much lower than the theoretical value of Richardson constant (32 A $\cdot \mathrm{cm}^{-2} \cdot \mathrm{K}^{-2}$ for p-type $\left.\mathrm{Si}\right){ }^{16}$ ) The deviation in the Richardson plot may be due to the spatially inhomogeneous barrier heights and potential fluctuations at the metalsemiconductor interface with low and high barrier areas. That is, the current through Ti/SOI Schottky contact flows preferentially through the lower barriers in the potential distribution. ${ }^{19-21)}$

It should be, however, noteworthy that the ideality factor and barrier height of Ti/SOI Schottky contact are strong function of temperature, as shown in Fig. 2. For instance, the barrier height increases with increasing temperature, while the increase in temperature leads to the decrease in ideality factor. At lower temperatures, the carriers do not have sufficient energy to surmount the high barrier. Therefore, the current transport is dominated by current flowing through the patches of lower Schottky barrier height, resulting in the higher ideality factor. As increasing the temperature, more carriers gain sufficient energy to surmount the higher barrier. ${ }^{19,22,23)}$ As a result, the current transport is dominated by the current that flows over the higher barrier and hence the ideality factor decreases at higher temperatures. This anomalous temperature dependence of the barrier height and ideality factor could be associated with the nature of inhomogeneous Schottky barrier consisting of a combination of low and high barrier patches with individual crosssectional areas, as suggested by many researchers. ${ }^{24-27)}$ Additionally, the variation in Schottky barrier height with temperature is fitted using following equation

$$
\Phi_{\mathrm{b}}(T)=\Phi_{\mathrm{bo}}(T=0)-\alpha T
$$

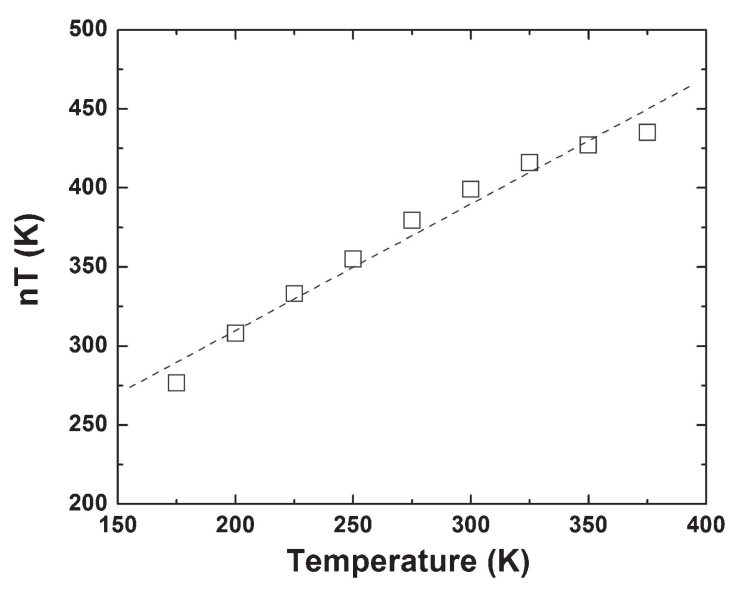

Fig. 4 Plot of $n T$ as a function of $T$ showing the $T_{0}$ anomaly of Ti/SOI Schottky contact.

where, $\Phi_{\mathrm{b}}(T)$ is the temperature dependent Schottky barrier height, $\Phi_{\text {bo }}(T=0)$ is the Schottky barrier height at $0 \mathrm{~K}$ and $\alpha$ is the temperature coefficient. The fit of eq. (6) to the data of Schottky barrier height shown in Fig. 2 yielded $\alpha=1.04 \times 10^{-3} \mathrm{~V} / \mathrm{K}$ and $\Phi_{\mathrm{b} 0}(T=0)=0.32 \mathrm{eV}$ in the temperature range of $175-375 \mathrm{~K}$. The barrier height values obtained increase with increasing temperature exhibiting a positive temperature coefficient which is inconsistent with the variation of the energy band gap with temperature that exhibits negative temperature coefficient. This discrepancy stems from the inhomogeneity of barrier height. ${ }^{28)}$ These inhomogeneities are explained by considering the Gaussian distribution of barrier heights that will follow later.

The temperature dependence of the ideality factor $n$ can be more understood by a plot of $n T$ versus $T$ as shown in Fig. 4. Generally, the temperature dependence of the ideality factor $n(T)$ has been frequently found to have the form of $n(T)=1+T_{0} / T$, where $T_{0}$ is a constant independent of temperature. ${ }^{1,21,29)} T_{0}$ is the measure of the temperature dependence of the ideality factor. From the linear fit to the $n T-T$ plot, the $T_{0}$ was calculated to be $150 \mathrm{~K}$, indicating the presence of the $T_{0}$ anomaly in the Ti Schottky contact to SOI. ${ }^{29)}$ Such parametric dependence is typical for a Schottky contact with a distribution of barrier inhomogeneities. Moreover, the deviation from linearity in a plot of $n T$ vs. $T$ could be associated with the high value of ideality factor since the ideality factor is governed by the current flow through the distribution of more low Schottky barrier height patches.

Figure 5 represents the plot of experimental barrier height versus the ideality factor for the Ti/SOI Schottky barrier diode for various temperatures. The least square fit of the experimental data in the plot indicated a linear relationship between the barrier height and the ideality factor. This may be attributed to lateral barrier inhomogeneities in Schottky diodes. ${ }^{30)}$ From the extrapolation of the experimental barrier heights versus ideality factor plot to $n=1$, a homogeneous barrier height is calculated to be $0.76 \mathrm{eV}$. According to Henisch, ${ }^{15}$ fluctuations in barrier heights are unavoidable, as they exist even in the most carefully fabricated systems. Thus, it can be hypothesized that the significant decrease 


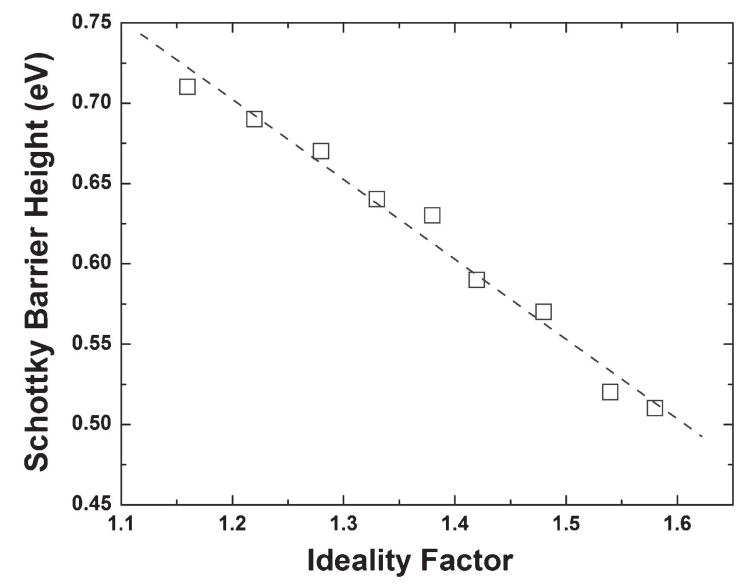

Fig. 5 Plot of the barrier height versus the ideality factor of Ti/SOI Schottky contact showing a linear correlation between these parameters.

in the barrier height and increase in the ideality factor of Ti/SOI Schottky contact, especially at low temperature may be due to the barrier inhomogeneities. A similar behavior was also observed by Schmitsdorf et al. ${ }^{30)}$ They found that a linear reduction of the effective barrier heights with increasing ideality factors in $\mathrm{Si}$ and $\mathrm{GaN}$ Schottky contacts could be attributed to lateral inhomogenous barrier heights.

As mentioned above, temperature dependent $I-V$ characteristics of Ti Schottky contact to SOI clearly reveal that its carrier conduction deviate from pure thermionic emission model. In order to quantitatively explain the non-ideal $I-V$ behavior of Ti/SOI Schottky contact, an analytical potential fluctuation model on spatially inhomogeneous Schottky barrier diodes was employed. The different types of barrier distribution function at the interface have been proposed by several researchers. ${ }^{20,23,27,31,32)}$ Among them, Gaussian distribution function has been widely used to describe the spatial variation of barrier heights in inhomogeneous Schottky diodes due to its simplicity, clear physical meaning and validity for small barrier inhomogeneity and moderate size of low-barrier patches. ${ }^{33,34)}$ In particular, it is well accepted to correlate the experimental data. ${ }^{35)}$ A spatial distribution of the barrier height at the metal-semiconductor interface of Schottky contacts caused by Gaussian distribution $P\left(\Phi_{\mathrm{b}}\right)$ with a standard deviation $\sigma_{0}$ around a mean barrier height $\overline{\Phi_{\text {bo }}}$ value was suggested by Werner and Guttler ${ }^{20,21)}$ and it is expressed by

$$
P\left(\Phi_{\mathrm{b}}\right)=\frac{1}{\sigma_{0} \sqrt{2 \pi}} \exp \left[-\frac{\left(\Phi_{\mathrm{b}}-\overline{\Phi_{\mathrm{bo}}}\right)^{2}}{2 \sigma_{\mathrm{o}}^{2}}\right]
$$

where the pre-exponential term is the normalization constant of the Gaussian barrier height distribution. The total current $I(V)$ across a Schottky contact containing barrier inhomogeneities at a forward bias can be expressed as

$$
I(V)=\int_{-\infty}^{+\infty} I\left(\Phi_{\mathrm{b}}, V\right) P\left(\Phi_{\mathrm{b}}\right) d \Phi_{\mathrm{b}}
$$

where $I\left(\Phi_{\mathrm{b}}, V\right)$ is the current for a barrier height $\Phi_{\mathrm{b}}$ at voltage $V$. By introducing $I\left(\Phi_{\mathrm{b}}, V\right)$ and $P\left(\Phi_{\mathrm{b}}\right)$ from eqs. (1) and (7) in eq. (8) the current of the Schottky barrier diode with the modified barrier can be expressed by

$$
\begin{aligned}
I(V)= & A A^{*} T^{2} \exp \left[-\frac{q}{k T}\left(\overline{\Phi_{\mathrm{bo}}}-\frac{q \sigma_{\mathrm{o}}^{2}}{2 k T}\right)\right] \exp \left(\frac{q V}{n_{\mathrm{ap}} k T}\right) \\
& \times\left[1-\exp \left(-\frac{q V}{k T}\right)\right]
\end{aligned}
$$

with

$$
I_{0}=A A^{*} T^{2} \exp \left(-\frac{q \Phi_{\mathrm{ap}}}{k T}\right)
$$

where $I_{0}$ is the observed saturation current, $\Phi_{\text {ap }}$ and $n_{\text {ap }}$ are the apparent barrier height and ideality factor, respectively. The $\Phi_{\text {ap }}$ and $n_{\text {ap }}$ are given by ${ }^{36,37)}$

$$
\begin{gathered}
\Phi_{\text {ap }}=\overline{\Phi_{\text {bo }}}-\frac{q \sigma_{\mathrm{o}}^{2}}{2 k T} \\
\left(\frac{1}{n_{\mathrm{ap}}}-1\right)=-\rho_{2}+\frac{q \rho_{3}}{2 k T}
\end{gathered}
$$

where $\rho_{2}$ and $\rho_{3}$ are voltage coefficients. Equation (9) is the form of eq. (1), but contains $\Phi_{\mathrm{ap}}$ and $n_{\mathrm{ap}}$ in the place of $\Phi_{\mathrm{b} 0}$ and $n$, respectively. Therefore, the fitting of experimental $I-V$ data using eq. (9) can still be carried out in the usual way. However, the values of $\Phi_{\text {ap }}$ and $n_{\text {ap }}$ derived should be interpreted in terms of eqs. (11) and (12), respectively. It is interesting to note that $\Phi_{\text {ap }}$ depends on the distribution parameters such as $\overline{\Phi_{\text {bo }}}$ and $\sigma_{\mathrm{o}}$ as well as temperature. In addition, it is obvious that the existence of $\sigma_{\mathrm{o}}$ leads to the decrease in $\Phi_{\text {ap }}$, which becomes more pronounced at lower temperatures. Figure 6 shows the plots of $\Phi_{\text {ap }}$ versus $1 / 2 k T$ and $\left(1 / n_{\text {ap }}-1\right)$ versus $1 / 2 k T$ for Ti/SOI Schottky diode. The linearity of both plots indicates the temperature dependent $I-V$ behavior of the Ti Schottky contact to SOI. This is in good agreement with the recent model which is related to thermionic emission over a Gaussian barrier height distribution. ${ }^{35-37)}$ From the intercept and slope of a linear fit to a plot of $\Phi_{\text {ap }}$ versus $1 / 2 k T$ (Fig. 6(a)), $\overline{\Phi_{\text {bo }}}$ and $\sigma_{\mathrm{o}}$ are extracted to be 0.87 and $0.11 \mathrm{eV}$, respectively. The $\sigma_{\mathrm{o}}$ is a measure of the barrier inhomogeneity. Namely, the Schottky diode with the best rectifying performance presents the best barrier homogeneity with a lower value of $\sigma_{\mathrm{o}}$. It should be noteworthy that the calculated value of $\sigma_{0}(0.11 \mathrm{eV})$ is significant, as compared to $\overline{\Phi_{\mathrm{bo}}}(0.87 \mathrm{eV})$. This implies the considerable existence of barrier inhomogeneity in Ti/SOI Schottky contact. The $\rho_{2}$ and $\rho_{3}$ depend on temperature and can be used to quantify the voltage deformation of the barrier height distribution. ${ }^{19)}$ The $\rho_{2}$ and $\rho_{3}$, extracted from the intercept and slope of linear fit of the plot of $\left(1 / n_{\mathrm{ap}}-1\right)$ versus $1 / 2 k T$ (Fig. 6(b)), respectively, are found to be -0.0171 and $-0.0127 \mathrm{~V}$ respectively. In particular, the linear behavior of the $\left(1 / n_{\text {ap }}-1\right)-1 / 2 k T$ plot suggests that the ideality factor does indeed express the voltage deformation of the Gaussian distribution of the barrier height. Although $\Phi_{\mathrm{b}}(T)$ in eq. (6) and $\Phi_{\mathrm{ap}}$ in eq. (11) are in the form of linear and inverse functions of temperature, respectively, they show the same temperature dependency $\left(\Phi_{\mathrm{b}}(T)\right.$ and $\Phi_{\text {ap }}$ increase with increasing temperature).

As indicated earlier, due to the lateral inhomogeneity of the Schottky barrier, the Richardson constant, extracted from a conventional Richardson plot (Fig. 3), was much lower than the theoretical Richardson constant. To obtain more reliable value of the Richardson constant by minimizing the effect 

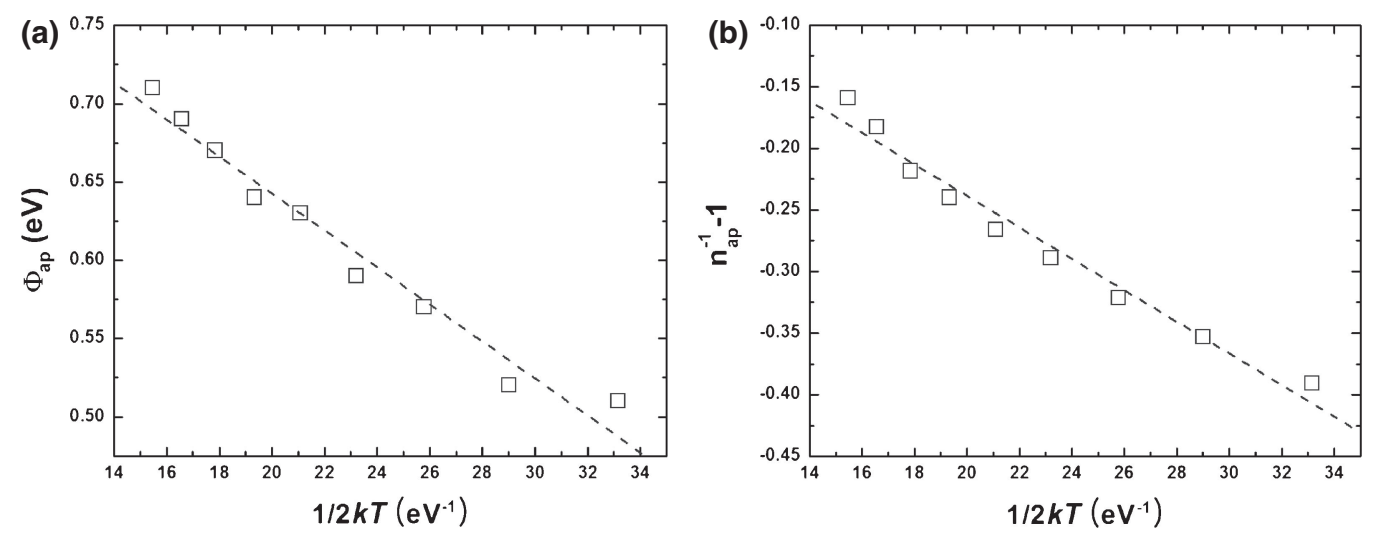

Fig. 6 Plots of (a) apparent barrier height and (b) apparent ideality factor versus $1 / 2 k T$ obtained from Ti/SOI Schottky contact.

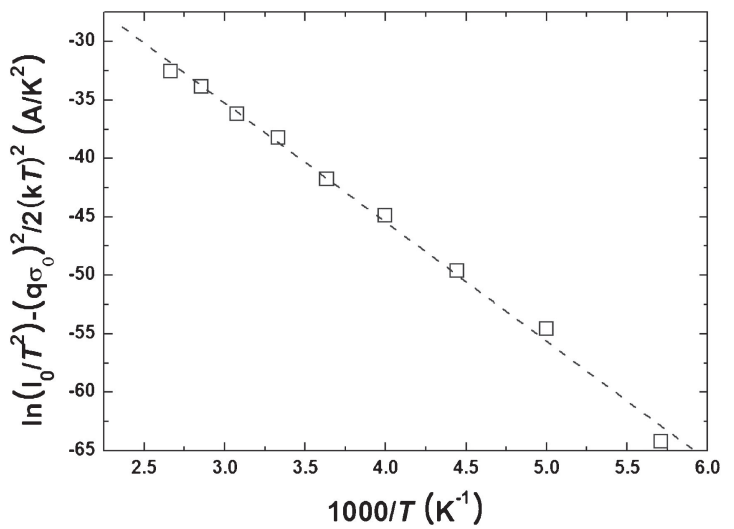

Fig. 7 Modified Richardson plot of $\ln \left(I_{0} / T^{2}\right)-q^{2} \sigma_{0}^{2} / 2 k^{2} T^{2}$ versus $1000 /$ $T$ for the Ti/SOI Schottky contact according to the Gaussian distribution of the barrier heights.

of Schottky barrier inhomogeneity on the $I-V$ characteristics of the Ti/SOI Schottky diode, we employed a modified Richardson plot (Fig. 7) using eq. (13) combined with eqs. (10) and (11) as following;

$$
\ln \left(\frac{I_{0}}{T^{2}}\right)-\left(\frac{q^{2} \sigma_{0}^{2}}{2 k^{2} T^{2}}\right)=\ln \left(A A^{*}\right)-\left(\frac{q \overline{\Phi_{\mathrm{bo}}}(T=0)}{k T}\right)
$$

A fit to the modified Richardson plot of $\ln \left(I_{0} / T^{2}\right)-\left(q \sigma_{0}\right)^{2} /$ $2(k T)^{2}$ versus $1000 / T$ should be a straight line from which the slope and $y$-axis intercept yield the mean Schottky barrier height $\overline{\Phi_{\mathrm{bo}}}(T=0)$ and the modified Richardson constant $A^{*}$ for a given diode area, respectively. From this plot, the mean Schottky barrier height and the modified Richardson constant are determined to be $0.87 \mathrm{eV}$ and $30.63 \mathrm{~A} \cdot \mathrm{cm}^{-2} \cdot \mathrm{K}^{-2}$ without using the temperature coefficient of the barrier heights, respectively. The mean Schottky barrier height extracted using the modified Richardson plot is identical to that using the plot of $\Phi_{\text {ap }}$ versus $1 / 2 k T$ (Fig. 6(a)). Furthermore, the modified Richardson constant is estimated to be $30.63 \mathrm{~A} \cdot \mathrm{cm}^{-2} \cdot \mathrm{K}^{-2}$. This value is very close to the theoretical value of p-type $\mathrm{Si}\left(32 \mathrm{~A} \cdot \mathrm{cm}^{-2} \cdot \mathrm{K}^{-2}\right)$. Therefore, the spatial inhomogeneity of the Schottky barrier heights cannot be neglected in the analysis of electrical measurements. In other word, the Gaussian distribution model is one of the possible models to explain the $I-V$ characteristics of the Ti/SOI Schottky system.

\section{Conclusions}

In the present work, the temperature-dependent $I-V$ measurements of Ti/SOI Schottky barrier diode were performed in the temperature range of $175-375 \mathrm{~K}$. The Ti/ SOI Schottky contact showed a considerable deviation from the ideal thermionic emission. With increasing temperature, the barrier height increased, whilst the corresponding ideality factor decreased. As considering the values of mean Schottky barrier height $(0.87 \mathrm{eV})$ and standard deviation $(0.11 \mathrm{eV})$ calculated using an analytical model with the Gaussian type of the barrier height distribution, the temperature-dependent behavior of the $I-V$ characteristics of the Ti/SOI Schottky contact could be associated with barrier height inhomogeneities at the Ti/SOI interface. A homogeneous barrier height extracted from the linear relationship between the experimental barrier heights and ideality factors was found to be $0.76 \mathrm{eV}$. Using the modified Richardson plot, the mean Schottky barrier height and the modified Richardson constant were obtained as $0.87 \mathrm{eV}$ and $30.63 \mathrm{~A} \mathrm{~cm}^{-2} \mathrm{~K}^{-2}$, respectively.

\section{Acknowledgements}

This work was supported by Priority Research Centers Program (2011-0031400) and Mid-Career Researcher Program (2010-0026523) through the National Research Foundation of Korea (NRF) funded by the Ministry of Education, Republic of Korea.

\section{REFERENCES}

1) M. E. Aydın, N. Yıldırım and A. Türüt: J. Appl. Phys. 102 (2007) 043701.

2) A. R. Arehart, B. Moran, J. S. Speck, U. K. Mishra, S. P. Denbaars and S. A. Ringel: J. Appl. Phys. 100 (2006) 023709.

3) S. Morikita and H. Ikoma: J. Vac. Sci. Technol. A 21 (2003) 226-233.

4) R. L. Ohta, C. E. Viana, N. I. Morimoto and B. H. V. Borges: J. Integr. Circuits Syst. 2 (2007) 85-88.

5) S. Kim, T. Shim and J. Park: J. Cer. Process. Res. 10 (2009) 507-511.

6) Y. Omura, A. Nakakubo and H. Nakatsuji: Solid-State Electron. 48 (2004) 1661-1666.

7) H.-K. Lim and J. Fossum: IEEE Trans. Electron Dev. 30 (1983) 1244 1251.

8) T. Ernst, S. Cristoloveanu, G. Ghibaudo, T. Ouisse, S. Horiguchi, Y. Ono, Y. Takahashi and K. Murase: IEEE Trans. Electron Dev. 50 (2003) 830-838. 
9) S. Chand and J. Kumar: Semicond. Sci. Technol. 10 (1995) 1680-1688.

10) K. C. Chiang, C. H. Cheng, K. Y. Jhou, H. C. Pan, C. N. Hsiao, C. P. Chou, S. P. McAlister, A. Chin and H. L. Hwang: IEEE Electron Dev. Lett. 28 (2007) 694-696.

11) I. Polishchuk, P. Ranade, T. J. King and C. Hu: IEEE Electron Dev. Lett. 22 (2001) 444-446.

12) E. H. Rhoderick and R. H. Williams: Metal-Semiconductor Contacts, 2nd ed., (Claredon Press, Oxford, 1988).

13) R. T. Tung: Mater. Sci. Eng. R 35 (2001) 1-138.

14) S. M. Sze: Physics of Semiconductor Devices, 2nd Ed., (John Wiley and Sons, New York, 1981).

15) H. K. Henisch: Semiconductor Contacts, (London, Oxford University, 1984).

16) H. Werner, U. Rau, J. F. Luy and P. Russer (Eds.): Springer Series in Electronics and Photonics, vol. 32, (Springer, Berlin, 1994).

17) C. Maleville, E. Neyret, L. Ecarnot, T. Barge and A. J. Auberton: IEEE International SOI Conference, (2001) pp. 19-20.

18) C. Maleville and C. Mazuré: Solid-State Electron. 48 (2004) 1055 1063.

19) R. T. Tung: Physical Review B 45 (1992) 13509-13523.

20) J. H. Werner and H. H. Guttler: J. Appl. Phys. 69 (1991) 1522-1533.

21) J. H. Werner and H. H. Guttler: J. Appl. Phys. 73 (1993) 1315-1319.

22) A. Gümüș, A. Türüt and N. Yalçin: J. Appl. Phys. 91 (2002) 245-250.

23) J. P. Sullivan, R. T. Tung, M. R. Pinto and W. R. Graham: J. Appl. Phys. 70 (1991) 7403-7424.
24) V. Janardhanam, Y.-K. Park, H.-J. Yun, K.-S. Ahn and C.-J. Choi: IEEE Electron. Dev. Lett. 33 (2012) 949-951.

25) H. C. Card and E. H. Rhoderick: J. Phys. D: Appl. Phys. 4 (1971) 1589-1601.

26) I. Jyothi, M.-W. Seo, V. Janardhanam, K.-H. Shim, Y.-B. Lee, K.-S. Ahn and C.-J. Choi: J. Alloy. Compd. 556 (2013) 252-258.

27) V. Janardhanam, A. Ashok Kumar, V. Rajagopal Reddy and P. Narasimha Reddy: J. Alloy. Compd. 485 (2009) 467-472.

28) M. Gökçen and M. Yıldırım: Chin. Phys. B 21 (2012) 128502.

29) J. D. Levine: J. Appl. Phys. 42 (1971) 3991-3999.

30) R. F. Schmitsdorf, T. U. Kampen and W. Monch: J. Vac. Sci. Technol. B 15 (1997) 1221-1226.

31) Y. P. Song, R. L. Van Meirhaeghe, W. H. Laflere and P. Cardon: SolidState Electron. 29 (1986) 633-638

32) N. Tugluoglu, S. Karadeniz, S. Acar and M. Kasap: Chin. Phys. Lett. 21 (2004) 1795-1798.

33) S. Acar, S. Karadeniz, N. Tugluoglu, A. B. Selcuk and M. Kasap: Appl. Surf. Sci. 233 (2004) 373-381.

34) S. Y. Zhu, R. L. Van Meirhaeghe, C. Detavernier, F. Cardon, G. P. Ru, X. P. Qu and B. Z. Li: Solid-State Electron. 44 (2000) 663-671.

35) M. K. Hudait, S. P. Venkateswarlu and S. B. Krupanidhi: Solid-State Electron. 45 (2001) 133-141.

36) M. K. Hudait and S. B. Krupanidhi: Phys. B 307 (2001) 125-137.

37) M. Sağlam, E. Ayyildiz, A. Gümüș, A. Türüt, H. Efeoğlu and S. Tüzemen: Appl. Phys. A 62 (1996) 269-273. 